\title{
Andreev reflection eigenvalue density in mesoscopic conductors.
}

\author{
P. Samuelsson ${ }^{a}$, W. Belzig ${ }^{b}$ and Yu. V. Nazarov ${ }^{c}$ \\ ${ }^{a}$ Département de Physique Theorique, Université de Genève, CH-1211 Genéve 4, Switzerland. \\ ${ }^{b}$ Department of Physics and Astronomy, Universität Basel, Klingelbergstr. 82, 4056 Basel, Switzerland. \\ ${ }^{c}$ Department of Nanoscience, TU Delft, Lorentzweg 1, 2628 CJ Delft, The Netherlands.
}

\begin{abstract}
The energy-dependent Andreev reflection eigenvalues determine the transport properties of normal-superconducting systems. We evaluate the eigenvalue density to get an insight into formation of resonant electron-hole transport channels. The circuit-theory-like method developed can be applied to any generic mesoscopic conductor or combinations thereof. We present the results for experimentally relevant cases of a diffusive wire and a double tunnel junction.
\end{abstract}

PACS numbers: 73.23.-b, 05.40.-a, 72.70.+m, 74.40.+k

Scattering theory provides a intuitive physical picture of transport in mesoscopic conductors [1, 2, [3]. A large number of transport properties are characterized by the transmission eigenvalues $\left\{T_{n}\right\}$ of the conductor, the eigenvalues of the matrix product $t t^{\dagger}$, where $t$ is the matrix of the transmission amplitudes and $n$ the channel index. Examples are the Landauer formula for the conductance $G \sim \sum_{n} T_{n}$, the shot noise power $P \sim \sum_{n} T_{n}\left(1-T_{n}\right)$ [4] or, more general, the full counting statistics $\sim \sum_{n} \ln \left[1+T_{n}(\exp (i \chi)-1)\right]$ [5]. Knowledge of the density of the transmission eigenvalues for a given mesoscopic conductor allows one to calculate the ensemble averaged transport properties. The transmission eigenvalue density has been derived for generic conductors such as diffusive wires [6, 11], chaotic cavities 7], double barrier junctions 8, 9], dirty tunnel barriers [10], or combinations thereof [9].

When a mesoscopic conductor is connected to a superconductor, the electronic properties of the conductor are modified by the induced proximity effect [12]. On a microscopic level, the proximity effect results from Andreev reflection at the normal-superconducting (NS) interface. In analogy to the normal conductor, the transport properties in the most interesting regime, at energies well below the superconducting gap, are naturally expressed in terms of the Andreev reflection eigenvalues $\left\{R_{n}\right\}$. The $\left\{R_{n}\right\}$ are the eigenvalues of the matrix product $s_{e h}^{\dagger} s_{e h}$, where $s_{e h}$ is the matrix of the Andreev reflection amplitudes. The conductance 13] $G_{N S} \sim \sum_{n} R_{n}$ and shotnoise power [14] $P_{N S} \sim \sum_{n} R_{n}\left(1-R_{n}\right)$ are the most investigated quantities.

Due to the dephasing of electrons and holes with an energy difference $2 E$, the Andreev reflection eigenvalues depend on energy on the scale of the Thouless energy $E_{c}$. Only at low energies $E \ll E_{c}$ are the Andreev reflection eigenvalues simply related [15] to the transmission eigenvalues as $R_{n}=T_{n}^{2} /\left(2-T_{n}\right)^{2}$, providing a direct relation between the density of Andreev reflection eigenvalues (ARED) and the density of transmission eigenvalues. To extend this relation to finite energies, one would need not only the correlations between transmission eigenvalues at different energies, but also the energy dependent

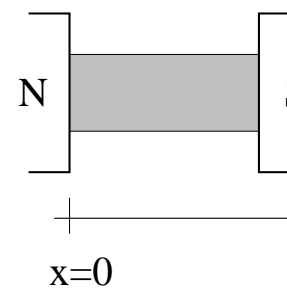

a)

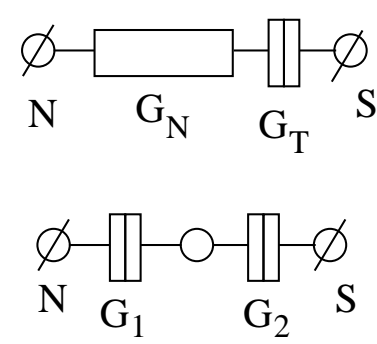

FIG. 1: Left: Schematic picture of a mesoscopic conductor connected to a normal $(\mathrm{N})$ and a superconducting (S) reservoir. Right: The circuit theory representation of two mesoscopic conductors: a) a diffusive wire in series with a tunnel barrier, b) two tunnel barriers in series. The conductances of the respective circuit elements are shown.

correlations between the transmission eigenvectors, i.e. the statistics of the whole energy dependent scattering matrix. Apart from being a complicated approach, this statistics is known only for a few generic conductors [1]. This makes it highly desirable to find a way to directly calculate the ARED, without going via the energy dependent statistics of the scattering properties of the normal conductor.

In this paper we present a theoretical approach to the energy dependent ARED for mesoscopic conductors. It comes in the form of a circuit theory for quasiclassical Green's functions [9, 11, 16]. The theory is applied to a diffusive wire junction, both with and without a tunnel barrier at the NS-interface, and to a double tunnelbarrier junction. In all cases, the induced proximity effect leads to an opening of electron-hole channels, $R_{n} \approx 1$, at energies of the order of $E_{c}$. Our results provide a unified and physically intuitive explanation for a large number of theoretical and experimental results on current and noise in NS-systems.

We consider a generic mesoscopic conductor with a large number of transverse channels $N \gg 1$ contacted to one normal and one superconducting reservoir (see Fig. (1). An electron, incident from the normal reservoir in channel $n$, has the amplitude $\left(s_{e h}\right)_{n m}$ to be backre- 
flected as a hole in channel $m$. To derive the ARED we follow Ref. 11] and define a function in terms of the Andreev scattering amplitudes (depending on energy) as

$$
H(E, \zeta)=\operatorname{Tr}\left[\frac{s_{e h} s_{e h}^{\dagger}}{1-\zeta^{2} s_{e h} s_{e h}^{\dagger}}\right]=\sum_{n} \frac{R_{n}}{1-\zeta^{2} R_{n}}
$$

which gives all moments $\operatorname{Tr}\left[\left(s_{e h} s_{e h}^{\dagger}\right)^{n}\right]$ when expanded in $\zeta$. From this function, the ARED $\rho(R, E)=\left\langle\sum_{n} \delta(R-\right.$ $\left.\left.R_{n}\right)\right\rangle$ is given as

$$
\rho(R, E)=\frac{-1}{\pi R^{2}}\left\langle\operatorname{Im}\left[H\left(E, \zeta=\frac{1}{\sqrt{R+i 0}}\right)\right]\right\rangle
$$

where $\langle\ldots\rangle$ denotes average over impurity (or sample) configurations. Next, the Andreev reflection amplitudes are related to the retarded anomalous Gorkov Green's function $F^{\dagger R}\left(\mathbf{x}, \mathbf{x}^{\prime}\right)$, constructed from the scattering state solutions [17 to the Bogoliubovde Gennes equation. Introducing $F_{n m}^{\dagger R}\left(x, x^{\prime}\right)=$ $\int d y d z d y^{\prime} d z^{\prime} \theta_{n}(y, z) \theta_{n}\left(y^{\prime}, z^{\prime}\right) F^{\dagger R}\left(\mathbf{x}, \mathbf{x}^{\prime}\right)$, with $\theta_{n}(y, z)$ the transverse wavefunction, we get

$$
\left(s_{e h}\right)_{n m}=-i \hbar \sqrt{v_{n} v_{m}} F_{n m}^{\dagger R}\left(x, x^{\prime}\right)
$$

with $F_{n m}^{\dagger R}\left(x, x^{\prime}\right)$ evaluated at the normal reservoir $(x=$ $x^{\prime}=0$ ) and where $v_{n}$ is the velocity in channel $n$. Using that $F^{A}\left(x^{\prime}, x\right)=-\left[F^{\dagger R}\left(x, x^{\prime}\right)\right]^{*}$, the trace becomes

$$
\operatorname{Tr}\left[s_{e h} s_{e h}^{\dagger}\right]=-\hbar^{2} \sum_{n, m} v_{n} v_{m} F_{n m}^{\dagger R}\left(x, x^{\prime}\right) F_{n m}^{A}\left(x^{\prime}, x\right) .
$$

Introducing the generalized quasiparticle current $I\left(\mathbf{x}, \mathbf{x}^{\prime}\right)=\sum_{n} \hbar v_{n} \theta_{n}(y, z) \theta_{n}\left(y^{\prime}, z^{\prime}\right) \delta(x) \delta\left(x^{\prime}\right)$, going from summation over modes to integration over transverse coordinates, we can write

$$
\begin{aligned}
\operatorname{Tr}\left[s_{e h} s_{e h}^{\dagger}\right] & =\int d \mathbf{x}_{1} d \mathbf{x}_{2} d \mathbf{x}_{3} d \mathbf{x}_{4} I\left(\mathbf{x}_{1}, \mathbf{x}_{2}\right) \\
& \times F^{R \dagger}\left(\mathbf{x}_{2}, \mathbf{x}_{3}\right) I\left(\mathbf{x}_{3}, \mathbf{x}_{4}\right) F^{A}\left(\mathbf{x}_{4}, \mathbf{x}_{1}\right) .
\end{aligned}
$$

As pointed out in Ref. [11], the right side of the expression can be evaluated at an arbitrary cross-section of the normal conductor. To obtain the traces of $s_{e h} s_{e h}^{\dagger}$ to all orders in Eq. (1), we introduce a $4 \times 4$-matrix Green's function in a fictitious $\zeta$-dependent field, coupling the advanced and retarded components as

$$
\begin{aligned}
\check{G}\left(\mathbf{x}_{1}, \mathbf{x}_{2}\right) & =\check{G}^{0}\left(\mathbf{x}_{1}, \mathbf{x}_{2}\right)+\int d \mathbf{x}_{3} d \mathbf{x}_{4} \check{G}^{0}\left(\mathbf{x}_{1}, \mathbf{x}_{3}\right) \\
& \times \zeta I\left(\mathbf{x}_{3}, \mathbf{x}_{4}\right)\left[\check{\tau}_{1}+\check{\tau}_{2}\right] \check{G}\left(\mathbf{x}_{4}, \mathbf{x}_{2}\right) .
\end{aligned}
$$

Here the unperturbed Green's function is

$$
\check{G}^{0}=\left(\begin{array}{cc}
\hat{G}^{R} & 0 \\
0 & \hat{G}^{A}
\end{array}\right), \quad \hat{G}^{R / A}=\left(\begin{array}{cc}
G^{R / A} & F^{R / A} \\
F^{\dagger R / A} & -G^{R / A}
\end{array}\right),
$$

suppressing coordinate dependence, and $\check{\tau}_{j}$ are matrices with the only nonzero element being $\left(\check{\tau}_{1}\right)_{42}=1$ and $\left(\check{\tau}_{2}\right)_{13}=-1$. With these definitions, it follows from the perturbation expansion in $\zeta$ that

$$
\int d \mathbf{x} d \mathbf{x}^{\prime} I\left(\mathbf{x}, \mathbf{x}^{\prime}\right) \operatorname{tr}\left[\check{\tau}_{1} \check{G}\left(\mathbf{x}, \mathbf{x}^{\prime}\right)\right]=\zeta H(E, \zeta),
$$

where the trace from now on is in the $4 \times 4$ matrix space. Starting from this expression, we can now apply the standard techniques for impurity averaged quasiclassical Green's functions, elaborated in detail for the circuit theory in Refs. 9, 16, 18]. This gives the relation

$$
\operatorname{tr}\left[\check{\tau}_{1} \check{I}(E, \zeta)\right] / G_{Q}=-i \zeta\langle H(E, \zeta)\rangle
$$

where $\check{I}(E, \zeta)$ is the energy dependent matrix current 16 through the junction and $G_{Q}=2 e^{2} / h$. As described in detail in Ref. 18, the terms in Eq. (6) containing $\zeta$ can be gauged away from the interior of the junction, giving rise to renormalized boundary conditions for the normal reservoir

$$
\check{G}_{N}=e^{i \zeta\left(\check{\tau}_{1}+\check{\tau}_{2}\right)} \check{G}_{N 0} e^{-i \zeta\left(\check{\tau}_{1}+\check{\tau}_{2}\right)}
$$

where $\hat{G}_{N 0}^{R / A}= \pm \hat{\sigma}_{z}$ with $\hat{\sigma}_{z}$ denoting a Pauli-matrix in Nambu space. The boundary conditions in the superconductor are not modified, i.e we have $\hat{G}_{S}^{R / A}=\hat{\sigma}_{x}$. We can then, within the circuit theory for matrix Green's functions Ref. [16], calculate the matrix current $\breve{I}(E, \zeta)$. Knowing $\check{I}(E, \zeta)$, the ARED can be obtained from Eq. (2) and (9) as

$\rho(R, E)=\frac{1}{G_{Q}} \frac{1}{\pi R^{3 / 2}} \operatorname{Re}\left\{\operatorname{tr}\left[\check{\tau}_{1} \check{I}\left(E, \zeta=\frac{1}{\sqrt{R+i \eta}}\right)\right]\right\}$.

This relation is the main technical result of the paper.

To illustrate the approach, we first consider a diffusive wire with conductance $G_{N}$ and length $L$ in good contact with a normal and a superconducting reservoir. This system is described by the quasiclassical Usadel equation [23] $D \partial_{x} \breve{G} \partial_{x} \breve{G}=-i E\left[\hat{\sigma}_{z}, \breve{G}\right]$, where $D$ is the diffusion coefficient. At low energies $E \ll E_{c}=\hbar D / L^{2}$, where the proximity effect is fully developed, the density is obtained directly from the transmission eigenvalue density of the wire [6], giving

$$
\rho_{\text {diff }}=\frac{G_{N}}{4 G_{Q}} \frac{1}{R \sqrt{1-R}} .
$$

This is just the same bimodal density as for the transmission eigenvalues [6], with a prefactor $1 / 2$. In the opposite, incoherent limit, $E \gg E_{c}$, the proximity effect is suppressed. It was recently shown [19] that in this limit, not only the current and the noise [20], but all moments of the current, can be found by mapping the wire onto an effective normal junction consisting of two diffusive wires in series. The same mapping procedure 

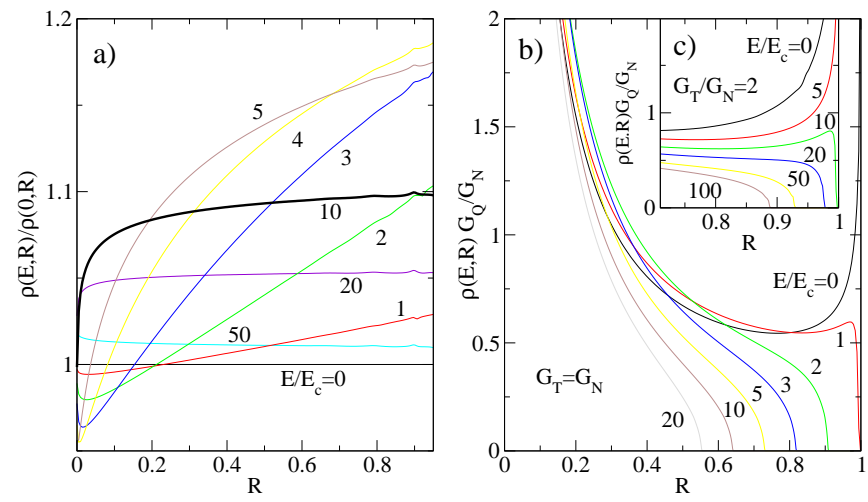

FIG. 2: Energy-dependent Andreev reflection eigenvalue densities (ARED) of diffusive contacts. a) ARED for a diffusive wire ideally connected to a normal and a superconducting reservoir. Energies are measured in units of the Thouless energy $E_{c}=\hbar D / L^{2}$ and the ARED is normalized to the curve for $E=0$. At energies around $E_{c}$, resonant electron-holes channels, $R \approx 1$, open up. b) ARED with a contact resistance $G_{T}=G_{N}$ at the NS-interface. For finite energies the ARED has an upper bound; there is a gap in the density for eigenvalues above a maximum eigenvalue $R_{\max }$. c) ARED with a contact resistance $G_{T}=2 G_{N}$ at the NS-interface. For $E \lesssim 10 E_{c}$ all eigenvalues contribute, whereas a gap opens up for higher energies.

can be used for the matrix Green's functions giving the ARED. As a result the ARED is just given by the transmission eigenvalue density of the effective junction (with conductance $G_{N} / 2$ and the replacement $T \rightarrow R$, yielding $\rho\left(R, E \gg E_{c}\right)=\rho_{\text {diff }}$, which is the same density as for low energies.

For energies of the order of $E_{c}$ the density differs from $\rho_{\text {diff. }}$ In this regime, the ARED was calculated numerically, following the prescription given in Ref. [21]. The result is shown in Fig. 2 (normalized to the ARED at $E=0$ ). Increasing the energy from zero, the density of open channels, $R \approx 1$, is enhanced in comparison to the density of closed channels, $R \approx 0$, until $E \approx 5 E_{c}$. For even higher energies, the density becomes qualitatively similar to $\rho_{\text {diff }}$ again, but with an overall enhancement [22]. The induced proximity effect thus results in an opening of electron-hole channels at energies of the order of $E_{c}$. This provides a complementary picture to the explanation [12] that an interplay of suppressed density of states and long-range pair-correlations leads to a modification of the transport properties of energies around $E_{c}$.

In particular, the opening of additional electron-hole channels provides a simple explanation to the reentrant conductance peak 24]. Moreover, the qualitative difference between the regimes $E \lesssim 5 E_{c}$ and $E \gtrsim 5 E_{c}$ explains the suppression of the effective charge $q_{\mathrm{eff}}(E)=$ $(4 / 3) e\left\langle\sum_{n} R_{n}\left(1-R_{n}\right)\right\rangle /\left\langle\sum_{n} R_{n}\right\rangle$ predicted theoretically in 21] and found experimentally in 25]. The overall enhancement of the ARED for $E \gtrsim 5 E_{c}$ cancels from the effective charge and explains why $q_{\mathrm{eff}}=2 e$ for $E \gtrsim 5 E_{c}$. In the regime of strong proximity $E \lesssim 5 E_{c}$ the enhancement of the density of open channels results, due to the factor $1-R_{n}$, in a lowering of $q_{\text {eff. }}$.

In the presence of a tunnel barrier $(I)$ at the NSinterface (see Fig. Th), it was found experimentally that due to the proximity effect the zero-voltage conductance is strongly enhanced [26] compared to what was expected from classical addition of resistances of the wire and the NIS-interface. This was explained in Ref. 27] as reflectionless tunneling. Backscattering by disorder in the wire enhances the chances for Andreev reflection and thus increases the conductance. Quantitatively, the ARED for a tunnel barrier with conductance $G_{T}$ at the NS-interface can be calculated numerically in the same way as without the barrier. In Fig. 20 we present the ARED for different energies in the case $G_{T}=G_{N}$. For low energies $E \ll E_{c}$ there is a finite fraction of completely open channels, $R=1$. Increasing the energy, the whole density shifts towards lower eigenvalues and the open channels start to close, i.e. there is a gap in the density above a maximum reflection eigenvalue $R_{\max }<1$. A similar behavior is seen for $G_{N}<G_{T}$, except that the opening of the gap occurs for a finite energy. In the example, shown in Fig. 25 for $G_{T}=2 G_{N}$, the gap opens for $E \gtrsim 10 E_{c}$. Note, that in the limit $G_{N} \ll G_{T}$ where the tunnel barrier can be neglected and there is a fraction of open channels for all energies. For a dominating tunnel barrier resistance, $G_{T}<G_{N}$, there are no completely open channels even for $E=0$. In the limit $G_{T} \ll G_{N}$ the shot-noise was recently observed experimentally [28] and studied theoretically [29]. A doubling of the full Schottky noise was found which can be understood by our results as a consequence of a concentration of the ARED at small $R$ for $G_{T} \ll G_{N}$.

As a second example, we investigate the ARED in a double tunnel-barrier junction (see Fig. П), a diffusive conductor with negligible resistance connected to one superconducting and one normal reservoir via tunnel barriers with conductance $G_{1}$ (to $N$ ) and $G_{2}$ (to $S$ ). The relevant energy scale for the proximity effect is now $E_{c}=\left(G_{1}+G_{2}\right) \delta /\left(4 G_{Q}\right)$, the inverse escape time from the diffusive region. Here $\delta$ is the level spacing in the normal region. For this system the ARED can be obtained analytically. The solution for the Green's function of the diffusive conductor for arbitrary energies is given in Refs. [30]. Eq. (11) gives after some algebra the ARED $\rho \equiv \rho(R, E)$

$$
\rho=\frac{G_{1}^{2} G_{2}^{2}}{2 \pi G_{Q} R^{5 / 4}}\left\{\begin{array}{lc}
\frac{1}{p \sqrt{p-\alpha \sqrt{R}}}, & 0<R<R_{I} \\
\frac{1}{q \sqrt{-q-\alpha \sqrt{R}}}, & R_{I}<R<R_{I I}
\end{array}\right.
$$

where $p=\left(4 G_{1}^{2} G_{2}^{2}-R \beta^{2}\right)^{1 / 2}, q=\left(R\left(\alpha^{2}+\beta^{2}\right)-\right.$ $\left.4 G_{1}^{2} G_{2}^{2}\right)^{1 / 2} \alpha=G_{1}^{2}+G_{2}^{2}-\left(E / E_{c}\right)^{2}\left(G_{1}+G_{2}\right)^{2}$ and $\beta=2 G_{1}\left(G_{1}+G_{2}\right) E / E_{c}$. The limiting eigenvalues are $R_{I}=4 G_{1}^{2} G_{2}^{2} /\left(\alpha^{2}+\beta^{2}\right)$ and $R_{I I}=4 G_{1}^{2} G_{2}^{2} / \beta^{2}$ (see Fig. 

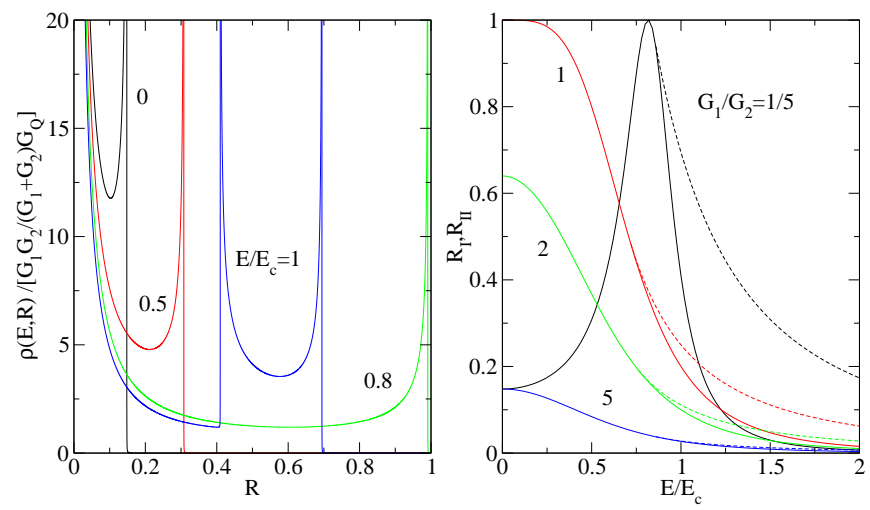

FIG. 3: Left panel: ARED for a double-barrier junction with $G_{2}=5 G_{1}$ for different energies $E / E_{c}$. Right panel: Limiting reflection eigenvalues $R_{I}$ (solid) and $R_{I I}>R_{I}$ (dashed) as a function of energy for different conductance ratios $G_{1} / G_{2}$

3), noting that the region $R_{I}<R<R_{I I}$ in the density in Eq. (13) is present only for $\alpha<0$.

The ARED is plotted in Fig. 3 for $G_{2}=5 G_{1}$, where the various properties of the ARED are clearly seen. For low energies $E<E_{c}\left(G_{1}^{2}+G_{2}^{2}\right)^{1 / 2} /\left(G_{1}+G_{2}\right)$, i.e. $\alpha>0$, the ARED is limited by an upper bound $R_{I}$ and is bimodal, see the upper term Eq. (13). In the limit $E \ll E_{c}$ the ARED reduces to the one obtained from the known [9] transmission eigenvalue density for the normal double tunnel-barrier junction. For higher energies, $E>E_{c}\left(G_{1}^{2}+G_{2}^{2}\right)^{1 / 2} /\left(G_{1}+G_{2}\right)$, the lower term in Eq. (13) contributes as well. This leads to a qualitatively new behavior of the ARED. The peak at the upper bound of the ARED splits and an extra bimodal band of Andreev reflection eigenvalues between $R_{I}$ and $R_{I I}$ emerges. Such a peculiar distribution with three singular peaks has to our knowledge not been observed before. We attribute this to a rigidity in position of the electron-hole resonaces in double barrier junctions, most pronounced for $G_{1} \ll G_{2}$. Upon changing the impurity configuration, each resonance fluctuates in energy between values related to the conductances $G_{1}$ and $G_{2}$. As a consequence, for energies below the lowest resonance, $\alpha>0$, the reflection eigenvalues take on values between 0 and $R_{I}$ and the density is bimodal. For higher energies, $\alpha<0$, the peaks at 0 and $R_{I}$ persist, but there is an additional peak at the maximum reflection eigenvalue $R_{I I}$ on resonance. In a junction dominated by impurity scattering, this rigidity effect is washed out.

There are two physically different regimes for the ARED, depending on the relation between the conductances $G_{1}$ and $G_{2}$. On one hand, for dominating coupling of the diffusive conductor to the normal reservoir, $G_{1}>$ $G_{2}$, the value of $R_{\max } \equiv \max \left(R_{I}, R_{I I}\right)$ never reaches unity, i.e. we have no completely open channels. This situation resembles the NIS-junction investigated above, i.e. the tunnel barrier to the normal reservoir plays the same role as the diffusive wire. On the other hand, for dominating coupling to the superconductor, $G_{2}>G_{1}$, there is always an energy, $E_{c} \sqrt{\left(G_{2}-G_{1}\right) /\left(G_{2}+G_{1}\right)}$, for which $R_{\max }$ reaches unity. Of particular interest is the limit $G_{2} \gg G_{1}$. In this limit, a proximity gap of magnitude $E_{c}$ is induced in the diffusive conductor. Only at energies very close to $E_{c}$, which is exactly at the edge of the induced gap, there are now open channels.

In conclusion, we have provided a theory for the Andreev reflection eigenvalue density in mesoscopic normalsuperconducting junctions. We find quite generally that the proximity effect leads to an opening up of resonant electron-hole channels at energies of the order of the Thouless energy $E_{c}$. This was exemplified by studying a diffusive wire and a double tunnel-barrier junction.

The work was supported by the Swiss network MaNEP (P.S.), the NCCR Nanoscience and the Swiss NSF (W. B.).

[1] C.W.J. Beenakker, Rev. Mod. Phys. 69, 731 (1997).

[2] Ya. M. Blanter and M. Büttiker, Phys. Rep. 3361 (2000).

[3] Quantum Noise in Mesoscopic Physics, edited by Yu. V. Nazarov (Kluwer, Dordrecht, 2003).

[4] M. Büttiker, Phys. Rev. Lett. 65, 2901 (1990).

[5] L.S. Levitov and G. B. Lesovik, Pis'ma Zh. Eksp. Teor. Fiz. 58, 225 (1993)].

[6] O.N. Dorokhov, Solid State Commun. 51, 381 (1984); A.D. Stone et al in B.L. Altshuler, P.A. Lee, R.A. Webb (Eds.), Mesoscopic Phenomena in Solids, North-Holland, Amsterdam (1991).

[7] H.U. Baranger and P.A. Mello, Phys. Rev. Lett. 73, 142 (1994); R.A. Jalabert, J.-L. Pichard and C.W.J. Beenakker, Europhys. Lett. 27, 255 (1994).

[8] J.A. Melsen and C.W.J. Beenakker, Phys. Rev. B 51 14483.

[9] Yu. V. Nazarov, in Quantum Dynamics of Submicron Structures, edited by H.A. Cedeira, B. Kramer, and G. Schön, NATO ASI Series E291 (Kluwer, Dordrecht).

[10] K.M. Schep and G.E.W. Bauer, Phys. Rev. Lett. 78, 3015 (1997).

[11] Yu. V. Nazarov, Phys. Rev. Lett. 73, 134 (1994).

[12] See e.g. W. Belzig et al, Superlatt. and Microstr. 25, 1251 (1999).

[13] C.J. Lambert, J. Phys. Condens. Matter 3, 6579; Y. Takane and H. Ebisawa, J. Phys. Soc. Jpn. 61, 1685 (1992).

[14] M.J.M. de Jong and C.W.J. Beenakker, Phys. Rev. B. 49, 16070 (1994).

[15] C.W.J. Beenakker, Phys. Rev. B. 46, 12841 (1992).

[16] Yu. V. Nazarov, Superlatt. Microstruct. 25, 1221 (1999).

[17] S. Datta, P.F. Bagwell, M.P. Anantram, Phys. Low-Dim. struct. 31 (1996).

[18] W. Belzig, in Ref. 3

[19] W. Belzig and P. Samuelsson, Europhys. Lett. 64, 253 (2003).

[20] K.E. Nagaev and M. Büttiker, Phys. Rev. B 63081301 (2001).

[21] W. Belzig and Yu. V. Nazarov, Phys. Rev. Lett. 87 
067006 (2001).

[22] The ARED is defined such that $G(E)=$ $2 G_{Q} \int d R \rho(E, R) R$ is the spectral conductance.

[23] K. D. Usadel, Phys. Rev. Lett. 25, 507 (1970).

[24] Yu. V. Nazarov and T.H. Stoof, Phys. Rev. Lett. 76, 823 (1996).

[25] B. Reulet et al., Phys. Rev. Lett. 90, 066601 (2003).

[26] A. Kastalsky et al., Phys. Rev. Lett. 67, 3026 (1991); P.H.C. Magnée et al, Phys. Rev. B. 50, 4594 (1994).

[27] B. van Wees, et al. Phys. Rev. Lett. 69510 (1992).
[28] F. Lefloch et al., Phys. Rev. Lett. 90, 067002 (2003).

[29] M.P.V. Stenberg and T.T. Heikkilä, Phys. Rev. B. 66, 144504 (2002). F. Pistolesi, G. Bignon, and F.W.J. Hekking, condmat/0303165, M. Houzet, F. Pistolesi, condmat/0310418.

[30] Yu. V. Nazarov and D.A. Bagrets, Phys. Rev. Lett. 88, 196801 (2002); J. Börlin, W. Belzig, and C. Bruder, ibid 88, 197001 (2002); P. Samuelsson, Phys. Rev. B 67 054508 (2003). 